\title{
Doppler assessment of the fetoplacental circulation in normal and growth-retarded fetuses
}

\author{
P.J.H.M. Reuwer, H.W. Bruinse, P. Stoutenbeek and A.A. Haspels \\ University Hospital Utrecht, The Netherlands \\ Accepted for publication 20 July 1984
}

\begin{abstract}
REUWER, P.J.H.M., BRUINSE, H.W., STOUTENBEEK, P. and HASPELS, A.A. (1984): Doppler assessment of the fetoplacental circulation in normal and growth-retarded fetuses. Europ. J. Obstet. Gynec. reprod. Biol., I8, 199-205.

The Pulsatility Index (PI) of Doppler flow signals from umbilical arteries was used to study flow resistance of the placental villous circulation. A preliminary reference curve of PI values in normal pregnancy was composed from 23 healthy women examined every $2 \mathrm{wk}$ from the 16 th postmenstrual week until delivery. The gestational age-related decrease of PI values reflects a reduction of flow resistance in the placental villous circulation.

In small-for-date fetuses significantly increased PI values were found, indicating that this simple technique may permit an early diagnosis of compromised fetoplacental circulation, even several weeks to months before fetal growth retardation is clınically presumed.
\end{abstract}

umbilical arteries: Pulsatility Index; flow resistance

\section{Introduction}

Clinical diagnosis of placental failure is a presumptive diagnosis usually based on signs of intra-uterine growth retardation (IUGR) and changes in fetal activity or heart rate patterns. By necessity, these signs are indirect and do occur late in the gradual process of impairing placental circulation with subsequent nutritive malfunction and ultimately deficient oxygen transfer.

Recent reports on Doppler flow recordings from umbilical arteries suggest that this non-invasive technique may provide direct information as to the functional state of the placental villous circulation (McCallum et al., 1978; Stuart et al., 1980; Giles et al., 1983; Reuwer et al., 1984). The method is based on the principle that the pulsatility of arterial flow reflects downstream vascular resistance (Gosling and King, 1974; Skidmore et al., 1980). The most accurate measure to quantify flow

Reprint requests: P.J.H.M. Reuwer, Department of Obstretics/Gynaecology, University Hospital Utrecht, Catharijnesingel 101, 3511 GV Utrecht, The Netherlands. 
pulsatility is the Pulsatility Index (PI), defined as the difference between systolic and diastolic peaks divided by the mean flow velocity (Fig. 1). A previous study (Reuwer et al., 1984) demonstrated the intra- and inter-observer reproducibility of PI values from umbilical arteries.

The aim of this pilot study was to establish reference PI values in normal pregnancy and to investigate whether deviating PI values could be related to IUGR.

\section{Subjects and methods}

Thirty-two Caucasian women with singleton pregnancies entered into the longitudinal study. Sixteen women were healthy primigravida and 8 multipara were low-risk, i.e., normal previous pregnancy and fetal outcome. The other 8 multiparous women were high-risk, i.e., previous infants with birthweights below the 10th percentile (Kloosterman (1970) birthweight tables). Postmenstrual age was confirmed by ultrasound measurements of the fetal crown-rump length in the first trimester. Doppler recordings from umbilical arteries were performed every 2 wk from the 16th until the 36 th postmenstrual week and weekly thereafter.

After delivery, 23 women (14 primigravida, 8 low-risk and 1 high-risk multigravida) fulfilled the required criteria for normal pregnancy: completely uneventful pregnancies and healthy term ( $>37 \mathrm{wk}$ ) infants without perinatal morbidity and with birthweights above the 10th percentile for gestational age.

Nine longitudinally studied women were excluded from the normal group; six patients ( 1 primigravida and 5 high-risk pregnancies) gave birth to small-for-date infants, defined as birthweights below the 10th percentile, and three women with infants above the 10th percentile were excluded for reasons of prematurity (1 primigravida) or hypertension ( 2 high-risk multigravida).

A second group of 20 pregnancies complicated by severe IUGR was studied. All birthweights in this group were below the 2.3 percentile. The first Doppler recordings in these patients were performed beyond 26 postmenstrual weeks after clinical diagnosis of IUGR was established.

Women were examined in the semi-recumbent position. Blood flow velocity signals from the umbilical arteries were recorded using a $4 \mathrm{MHz}$ pulsed Doppler device (ALVAR). The Doppler recording procedure and extensive instrumental specifications, as well as the adequacy of the signal processing technique by zero-cross detection, have been previously described (Reuwer et al., 1984). A

Pulsatility Index (PI)

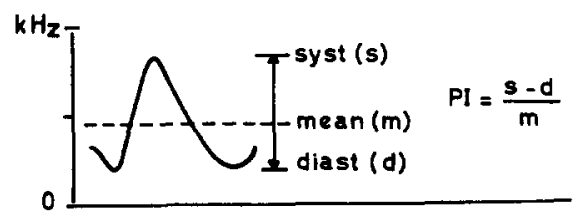

Fig. 1. The Pulsatility Index defined by Gosling and King (1974). The PI is independent of the angle between the artery and the ultrasound beam and therefore allows comparisons between several examinations and between patients. 
recording was accepted for analysis if a steady state of at least 5 heart beats had been obtained (Fig. 2). Although fetal movements disturbed steady state quite often, at least 3 or more appropriate recordings could be made most often within 25 minutes. To minimize selection bias, consistently those 3 feasible parts were analysed with the highest mean velocity. Systolic, diastolic and mean velocity values, visually averaged over 5 heart beats, were read from the paper strip and the Pulsatility Index (Fig. 1) was calculated. The PI value of an examination was taken as the average of the PIs of the 3 selected recordings.

The slope of the reference PI curve with advancing pregnancy was statistically tested against zero; 4 consecutive PI values of each woman were taken to calculate a least-square regression line. The slope (averaged slopes of 23 normal pregnancies) was tested against zero using the Student $t$-test (level of significance $P<0.05$ ).

\section{Results}

In the first half of normal pregnancy umbilical arterial blood flow mostly returned to the zero-line during diastole (i.e., below the $200 \mathrm{~Hz}$ high-pass filter). An example is shown in Fig. 2, left. Beyond the 22th postmenstrual week there was always a continuous perfusion of the placenta (example Fig. 2, right). The PI decreased significantly with advancing normal pregnancy (Fig. 3, left), mainly due to an increase in diastolic and thus mean velocity level. The PI decrease in normal gestation was most marked before the 28th postmenstrual week (Fig. 3, left). In the last trimester, the PI decreased more gradually but still significantly up to the 38th wk. An example of the course of PI values in a normal pregnancy is shown in Fig. 3, right. Reference values were taken as the mean PI \pm 2 S.D. of the normal pregnancies.

All fetuses with severe IUGR had increased PI values more than 3 standard deviations above normal, except for 1 infant having normal PI values between 0.9 and 1.0 from the 29 th to the 31 th postmenstrual week. This exceptional infant

UMBILICAL ARTERY DOPPLER SHIFT
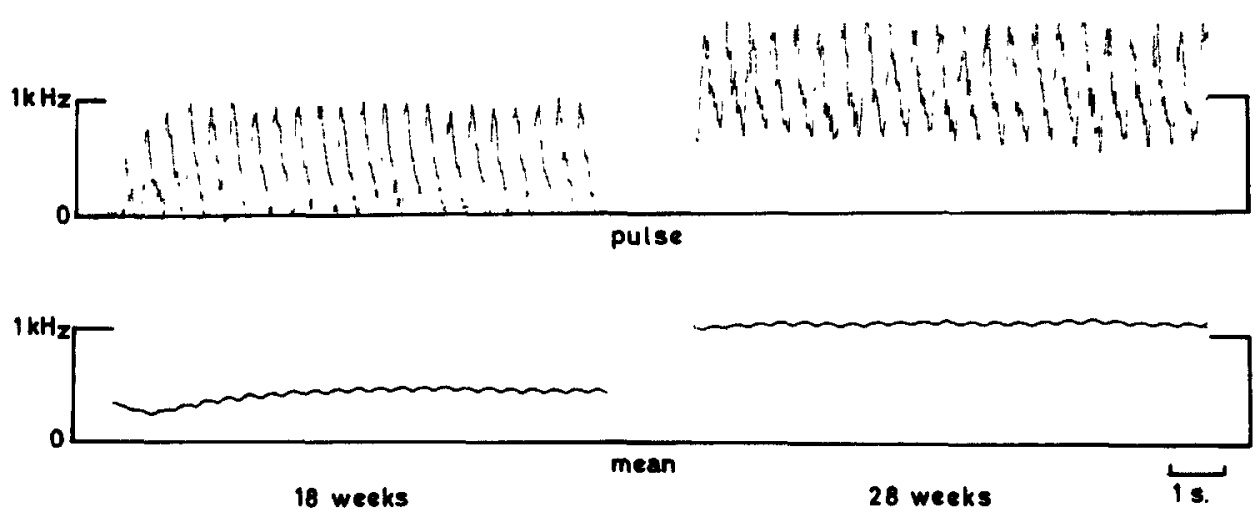

Fig. 2. Doppler recordings at 18 and 28 postmenstrual weeks in normal pregnancy. PI values are 2.1 and 0.9 , respectively. 

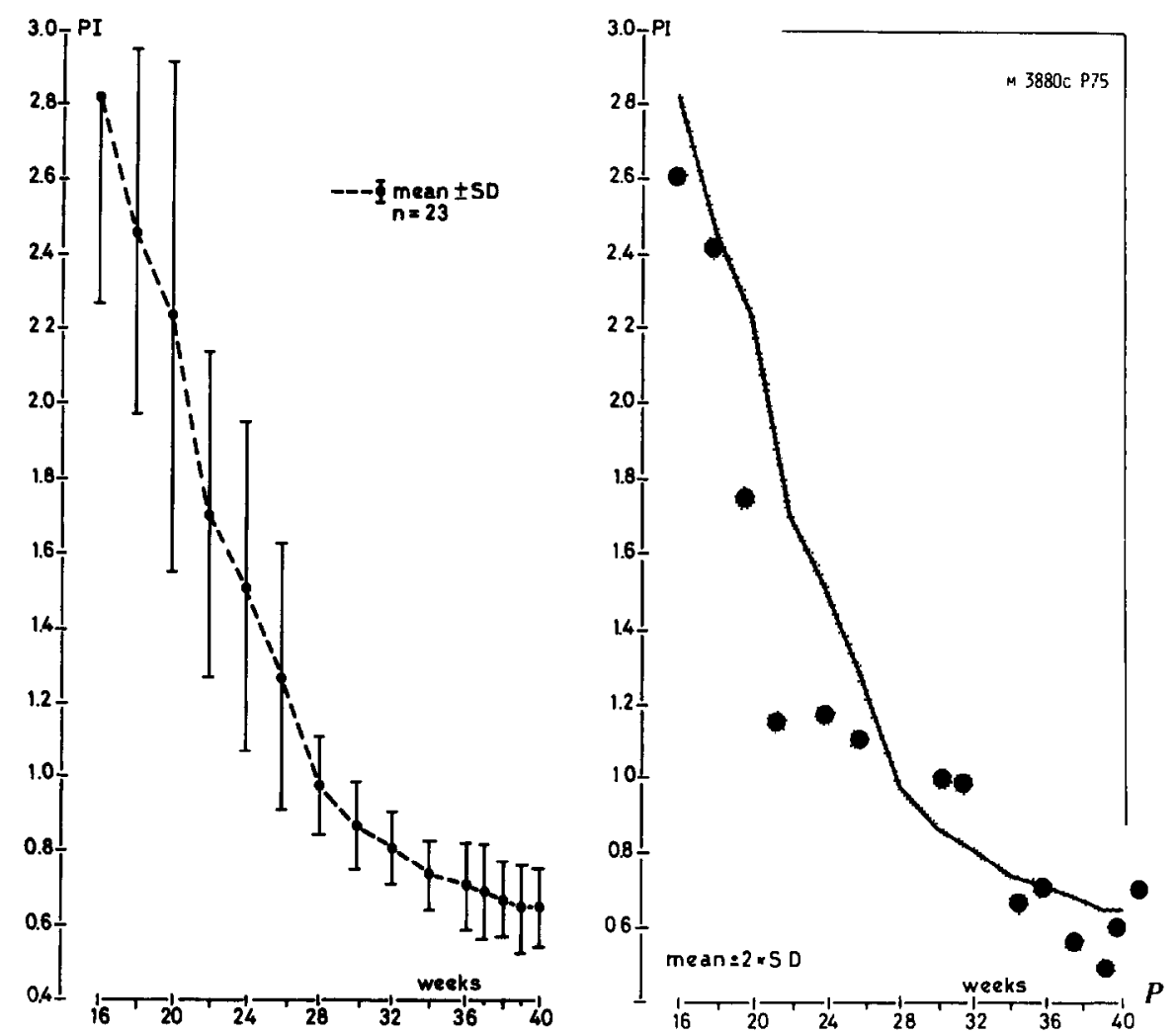

Fig. 3. Left panel: mean PI values in normal pregnancy. Right panel: example of a normal fetus. In the upper right corner, sex, birthweight and percentile of the infant. $P$ on the absciss stands for Parturition.

UMBILICAL ARTERY DOPPLER SHIFT

28 weeks
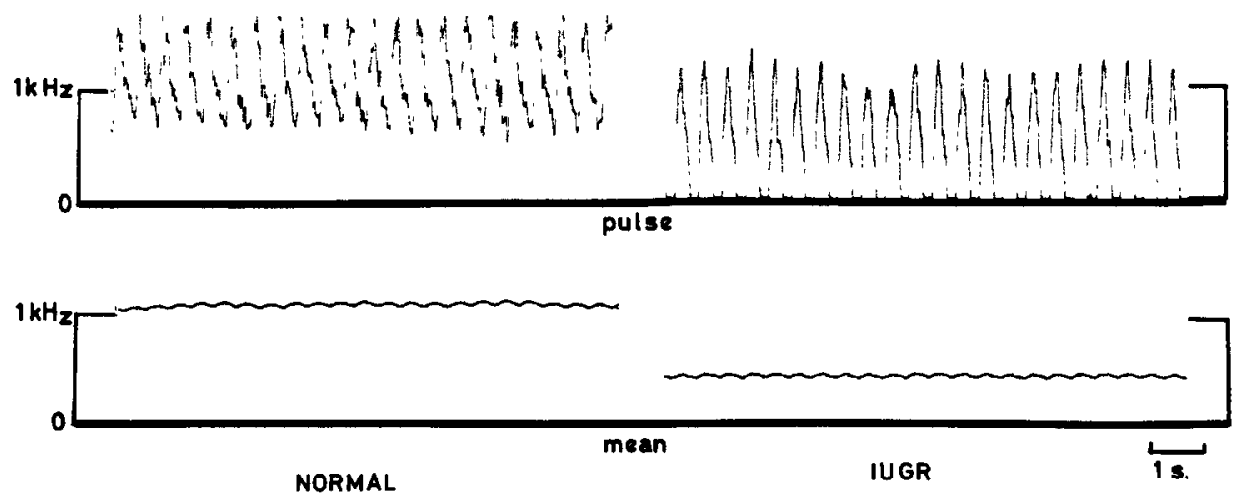

Fig. 4. Doppler recordings at $28 \mathrm{wk}$ in a normal and in a severely complicated pregnancy. 
turned out to be severely malformed (triploidy) with, however, a completely normal placenta. The other 19 fetuses with severe IUGR had no congenital malformations but evident placental pathology. The increased PI values in these cases were mainly caused by relative low or even absent diastolic velocity levels. An example is shown in Fig. 4, right.

Five out of the 6 longitudinally studied patients, giving birth to small-for-date infants (below the 10th percentile), had serially, and 1 had alternately, increased PI values more than 2 standard deviations above normal. PI values were increased several weeks to months before suboptimal fetal growth was clinically suspected. Two examples are shown in Fig. 5. In the patient presented in Fig. 5 left, suboptimal fetal growth was first suspected at 37 wk (fundal palpation and sonographic fetal abdomen area $<2.3$ percentile), whereas PI values were increased 8 wk earlier. Microscopical examination of the placenta revealed assymmetrical maturation failure. In the patients presented in Fig. 5 right, suboptimal fetal growth was not presumed at all on the basis of serial routine examinations (fundal palpation), whereas PI
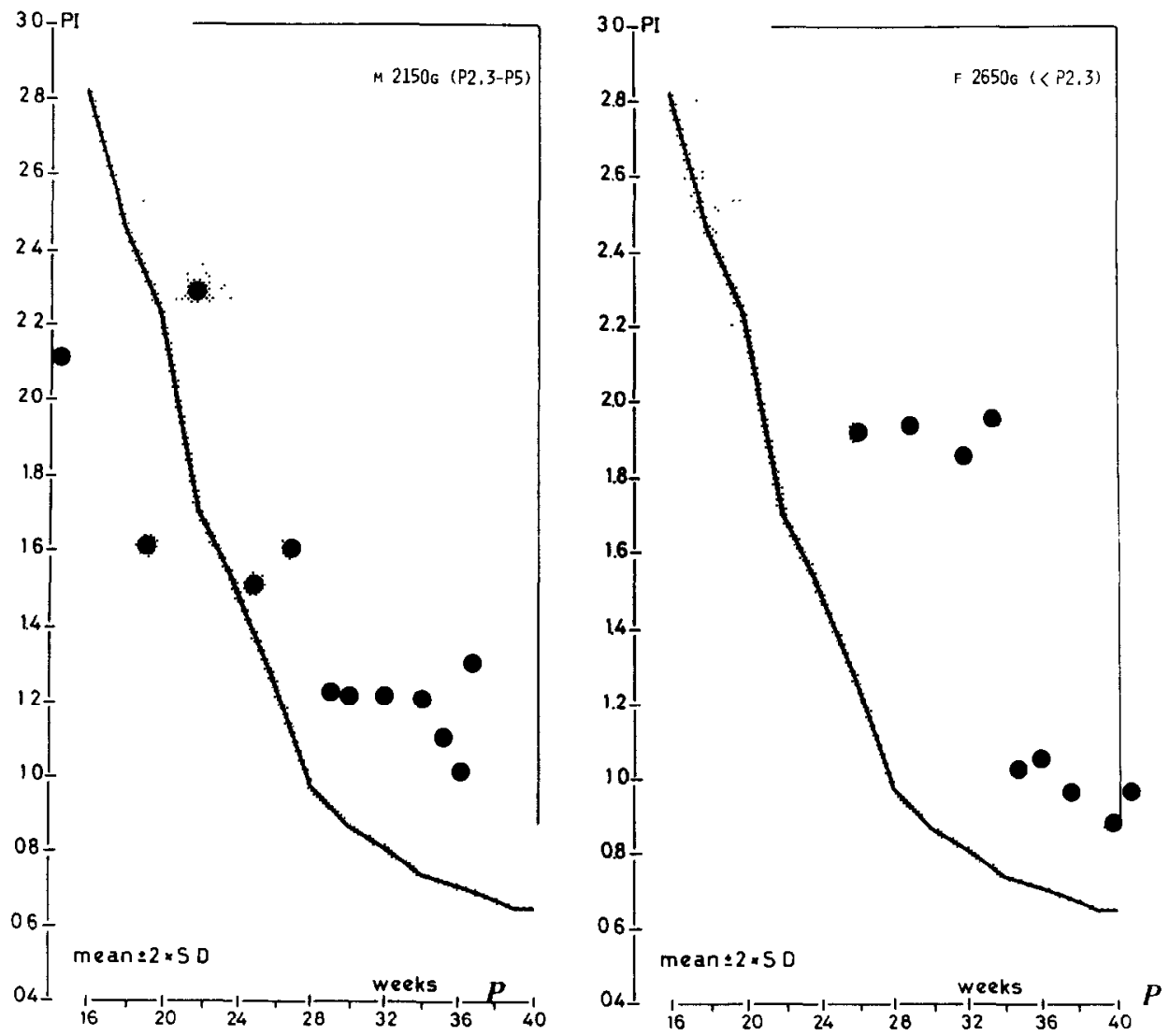

Fig. 5. Two examples of early increased PI values in small-for-date infants. 
values were increased as early as the 28 th postmenstrual week. Several old infarctions were found in the placenta.

In the group of 23 pregnancies defined as normal, 2 patients had serially increased PI values. Both infants had birthweights between the 10th and 25th percentile. One of these infants had a circumvallate placenta.

\section{Discussion}

The possibility to study the vascular resistance of an organ by examining the flow pulsatility of its arterial supply has been established for several vascular beds in adults (Gosling and King, 1974; Atkinson and Wells, 1977; Skidmore et al., 1980).

The PI of the umbilical arteries decreased significantly with advancing normal pregnancy (Fig. 3), reflecting a reduction in fetoplacental flow resistance. This reduction in flow resistance is presumably caused by ripening of the placental villous system. The intra-individual course of PI values is rather saltatory (Fig. 3, right), as might be expected from a hemodynamic variable. The intra-individual biological variations per examination have been previously described (Reuwer et al., 1984).

A different way of quantifying blood flow pulsatility by means of the $\mathrm{A} / \mathrm{B}$ ratio (the ratio of systole to end-diastole) was reported by Stuart et al. (1980), yielding likewise decreasing values. We prefer the PI to the A/B ratio for reasons of mathematical accuracy. Firstly, the denominator of the PI (mean velocity) is higher than the denominator of the A/B ratio (end-diastolic velocity), making the PI less sensitive to small reading errors. Secondly, end-diastolic flow may return to zero in the first half of normal pregnancy (Fig. 2) or in the second half in cases of complicated pregnancies (Fig. 4), rendering (alternately) infinite $A / B$ ratios. This prevents averaging of $\mathrm{A} / \mathrm{B}$ ratios of several steady recordings.

The gestational age-related decline of PI values is most marked before the 28th postmenstrual week (Fig. 3). This is in accordance with data from invasive lamb studies where the gestational age-related increase in umbilical blood flow is initially achieved mainly by a fall in vascular resistance and in the later part relatively more by a rise in fetal arterial pressure (Boddy, 1979).

In all fetuses with severe IUGR due to placental failure, PI values were extremely elevated, mainly caused by relatively low or even absent end-diastolic velocity levels (Fig. 4). The end-diastolic flow reduction as such actually indicates increased peripheral resistance (McDonald, 1974). Giles et al. (1983) reported increased flow pulsatility (A/B ratio) in high-risk pregnancies and related these findings to placental microvascular pathology. They concluded that the pulsatility of umbilical artery flow correlates well with arteriolar obliteration in the tertiary stem villi. These and our observations suggest that umbilical PI alterations do reflect changes in placental villous resistance. However, the numerical correlations still have to be established in future basal experiments. Computer simulation studies preceding animal experiments are now in progress.

Meanwhile, the present observations demonstrate that this Doppler method does distinguish accurately severe placental circulatory failure from normal placental function. All 19 cases of severe placental failure (IUGR) were antenatally recognized. Of even more clinical interest is the antenatal recognition of normal PI values 
in the severely growth-retarded triploidic infant with a normal placenta. In addition, the present results suggest that the PI may detect a suboptimal placental circulation at an early stage, several weeks to months before fetal compromise is clinically presumed on the basis of current diagnostics (Fig. 5). Although no general conclusions on the diagnostic value can be reached by these preliminary observations, the results suggest that the PI shows promise as a simple and clinically useful tool for early diagnosis of impaired fetal placental circulation. Moreover, the method may enhance our understanding of the subject of placental failure by distinguishing vascular from non-vascular causes. The present results justify an extension of the study to establish definitive PI reference values and to determine its diagnostic significance for the early detection of placental failure.

\section{Acknowledgement}

The authors thank Miss. M. Zwinkels for her skillful assistance.

\section{References}

Atkınson, P. and Wells, P.N.T. (1977): Pulsed Doppler ultrasound and its clinical application. Yale J. Biol. Med., 50, 367-373.

Boddy, K. (1979): Fetal blood flow to and from the placenta. In: Placental Transfer, pp. 45-59. Editors: G.V.P. Chamberlain and W.W. Wilkinson. Pitman Medical, London.

Giles, W.B., Trudinger, B.J. and Baird, P.J. (1983): Antenatal assessment of placental flow resistance and placental microvascular anatomy. 10th Conference on Fetal Breathing, Movements and other Fetal Measurements. Malmö, Sweden, June 6-8 (abstract).

Gosling, R.G. and King, D.H. (1974): Continuous wave ultrasound as an alternative and complement to $\mathrm{X}$-rays in vascular examinations. In: Cardiovascular Applications of Ultrasound, pp. 266-282. Editor: R.S. Reneman. North-Holland/American Elsevier, New York.

Kloosterman, G.J. (1970): On intrauterine growth. Int. J. Obstet. Gynaec., 8, 895-912.

McCallum, W.D., Williams, C.S., Napel, S. and Daigle, R.E. (1978): Fetal blood velocity waveforms. Amer. J. Obstet. Gynec., 132, 425-429.

McDonald, A.D. (1974): Blood Flow in Arteries. 2nd Edn. Edward Arnold, London.

Reuwer, P.J.H.M., Nuyen, W.C., Beijer, H.J.M., Heethaar, R.M., Bruinse, H.W., Stoutenbeek, P. and Haspels, A.A. (1984): Characteristics of flow velocities in the umbilical arteries, assessed by Doppler ultrasound, Europ. J. Obstet. Gynec. reprod. Biol., 17, 397-408.

Skidmore, R., Woodcock, J.P., Wells, P.N.T., Bird, D. and Baird, R.N. (1980): Physiological interpretation of Doppler shift waveforms. III, Clinical results. Ultrasound Med. Biol., 6, 227-231.

Stuart, B., Drumm, J., FitzGerald, D.E. and Duignan, N.M. (1980): Fetal blood velocity waveforms in normal pregnancy. Brit. J. Obstet. Gynaec., 87, 780-785. 